\title{
INFINITESIMAL BENDING AND TWISTING IN ONE-DIMENSIONAL DYNAMICS
}

\author{
FREDERICK P. GARDINER
}

\begin{abstract}
An infinitesimal theory for bending and earthquaking in onedimensional dynamics is developed. It is shown that any tangent vector to Teichmüller space is the initial data for a bending and for an earthquaking ordinary differential equation. The discussion involves an analysis of infinitesimal bendings and earthquakes, the Hilbert transform, natural bounded linear operators from a Banach space of measures on the Möbius strip to tangent vectors to Teichmüller space, and the construction of a nonlinear right inverse for these operators. The inverse is constructed by establishing an infinitesimal version of Thurston's earthquake theorem.
\end{abstract}

\section{INTRODUCTION}

A smooth curve of quasisymmetric homeomorphisms of a circle induces a smooth curve in Teichmüller space. An important problem in dynamics is to construct such a curve which is compatible with a dynamical system (see, for example, [16] and [17]). One method of construction comes from the existence theorem in the complex domain for solutions $h(z)$ to the Beltrami equation $h_{\bar{z}}(z)=\mu(z) h_{z}(z)$. If the Beltrami coefficient $\mu$ is compatible with a twodimensional dynamical system, such as a Fuchsian or Kleinian group or an expanding polynomial-like mapping defined in a neighborhood of a repeller, then the homeomorphism $h$ restricted to the limit set conjugates the dynamical system to another equivalent dynamical system. The compatibility of the Beltrami coefficient is what forces the new dynamical system to act on the limit set as a group of Möbius transformations or as an expanding polynomial-like mapping. But in order to make use of the Beltrami coefficient, one must be able to extend the dynamical system to the complex domain.

Thurston's earthquake theorem [18] is another way to construct dynamical curves in Teichmüller space. Although the earthquake itself takes place along nonintersecting geodesics in the hyperbolic plane, it is given by a measure $\sigma$ defined on the direct product of the boundary of the hyperbolic plane with itself. The measure $\sigma$ defined on $S^{1} \times S^{1}$ takes the place of the Beltrami coefficient $\mu$, and the compatibility of $\sigma$ with a real dynamical system can be expressed without extending the dynamical system to the complex plane. It is an idea of Dennis Sullivan that such $\sigma$ can be used to construct curves of

Received by the editors April 23, 1993 and, in revised form, May 18, 1994.

1991 Mathematics Subject Classification. Primary 32G15, 34A34, 43A75.

Partially supported by the National Science Foundation, DMS 9204069-001. 
one-dimensional dynamical systems in a given smoothness class. In this paper we set up the infinitesimal theory for the construction of such curves.

A typical simple bending homeomorphism is given by the formula

$$
h(x)=|x|^{\alpha} \operatorname{sign}(x), \quad 0<\alpha \text { and } x \text { in } \mathbb{R} .
$$

Our justification for calling this a bending is that it arises as the welding homeomorphism for the real axis in the complex plane bent in the following way. The positive real axis is fixed and the negative real axis is bent to a ray emanating from the origin and pointing with angle $\pi \beta, 0<\beta<2$. Then $f(z)=z^{\beta}$ is the Riemann mapping from the upper half plane onto the upper side of this bent real axis, normalized to fix 0,1 , and $\infty$. Moreover $g(z)=z^{\gamma}$ is the similarly normalized Riemann mapping from the lower half plane to the lower side of this bent real axis, where $\beta+\gamma=2$. One finds that the welding homeomorphism, which is $g^{-1} \circ f$ restricted to the real axis, is equal to $h$, where $\alpha=\beta /(2-\beta)$.

A typical simple earthquake homeomorphism $h$ is given by the formula

$$
h(x)= \begin{cases}c x & \text { for negative } x, c \text { in } \mathbb{R}_{+}, \\ x & \text { for nonnegative } x .\end{cases}
$$

The rationale for calling this $h$ a simple earthquake is that it is the boundary value of the self-mapping of the upper half plane which is the identity in the first quadrant and $z \mapsto c z$ in the second quadrant. Of course, this mapping is discontinuous along the imaginary axis and, if $c>1$, it is called a right earthquake because, for a person standing in the first quadrant and looking across the $y$-axis, the second quadrant appears to move to the right. Similarly, for a person standing in the second quadrant and imagining himself to be stationary, the first quadrant would appear to move also to the right. The $y$-axis is the hyperbolic geodesic which is the fault line for this simple earthquake.

The first important idea of this paper is the relationship between bending, earthquaking and the Hilbert transform. In sections 1, 2, and 3 we show why the Hilbert transform of an infinitesimal bend is precisely equal to an infinitesimal earthquake. This relationship is suggested but not explicitly declared in the paper of Wolpert [19] on the Fenchel-Nielsen twist deformations.

The observation that the classical Hilbert transform gives the almost complex structure for Teichmüller space is due to Kerckhoff. In [13] Nag and Verjovsky make the connection between the almost complex structure for Teichmüller theory and the almost complex structure for the Lie algebra of the Lie group of the $C^{\infty}$ diffeomorphisms of $S^{1}$. In the Ahlfors-Bers approach to Teichmüller theory, the holomorphic dependence of a solution to the Beltrami equation on the Beltrami coefficient $\mu$ leads to the complex structure for Teichmüller space. In a purely one-dimensional theory, we expect the relationship between bending and earthquaking to be the foundation for a complex structure on a deformation space.

The second idea of this paper is to introduce a suitable Banach space norm for measures on $S^{1} \times S^{1}$ and to show that the infinitesimal bending operator gives a bounded linear mapping from this space to the Banach space to the space $\mathscr{Z}$ of Zygmund bounded vector fields.

The third idea is the construction of a right inverse to the bending operator, the existence of which implies that the infinitesimal bending operator is surjec- 
tive. Since the Hilbert transform of an infinitesimal bend is an infinitesimal earthquake, it suffices to construct a right inverse to the infinitesimal earthquake operator. The construction we give arises from the study of a version of Thurston's earthquake theorem for finite configurations of points on the unit circle. So far as we can see, this version of the earthquake theorem cannot be deduced from Thurston's result for homeomorphisms of $S^{1}$. The version we have in mind applies to the following initial data. A finite subset $E$ of $S^{1}$ is given and a cyclic order preserving mapping $h$ from $E$ to $h(E) \subset S^{1}$ is also given. Then there exists a unique allowable way to draw hyperbolic fault lines connecting the points of $E$ and to assign nonnegative weights to these lines so that, up to postcomposition by a Möbius transformation, the mapping $h$ is realized by sliding to the left along each of the hyperbolic lines a hyperbolic distance equal to the assigned weights. The configuration of fault lines is allowable if no two of the lines intersect and no two adjacent points of $E$ are joined by a line. The restrictions on allowable configurations of fault lines are key to understanding the construction; without them and the assumption that the weights are nonnegative, the homeomorphism fails to be uniquely determined. For the mapping $h$ defined only on the finite set $E$, the construction gives a canonical way to select a homeomorphism of the whole circle which agrees, up to postcompositions by a Möbius transformation, with $h$ on $E$. On each of the intervals between the points of $E$ the homeomorphism is a canonically selected Möbius transformation. Thurston's earthquake theorem applied to this specifically selected homeomorphism will be realized by an earthquake along a lamination by ideal polygons (which generically are ideal triangles) such that the sides of these polygons are the fault lines of the allowable configuration.

We use an infinitesimal version of this theorem to construct a right inverse to the infinitesimal earthquake operator. For finite configurations the construction is carried out by taking a lower quadratic polynomial convex hull. To obtain the general solution, we use a limiting process taking limits in the weak topology on $\mathscr{Z}$.

The graph of a simple infinitesimal earthquake is a piece of a concave parabola truncated below by a horizontal line. This basic element is closely related to the theory of wavelets. The first derivative of the simple infinitesimal earthquake is identically zero except on an interval, where it is linear and has mean equal to zero. The construction we give for Lemma 5.1 gives an effective way to encode in an atomic measure a vector field defined on a finite set $E$. The vector field decomposes in a unique way into a nonnegative sum of these wavelets with nonoverlapping intervals of support. By appropriate applications of the Hilbert transform, we also can decompose a general Zygmund bounded vector field into bending wavelets. The formulas for the bending wavelets are $B(x)=x \log |x|$ or the pullback of $B(x)$ by a Möbius change of coordinates. Since the derivative of $B(x)$ is essentially $\log |x|$, we expect there is a canonical way to decompose a BMO-function into Möbius pullbacks of the function $\log |x|$ which fits with the John-Nirenberg theorem [8, p. 230]. The John-Nirenberg theorem characterizes BMO-functions by exponential decay of distributions at all scales.

The consequence of the three ideas is that there are a bending measure and an earthquake measure for any vector field in $\mathscr{Z}$. Since these measures can be transported by pushforward by an arbitrary quasisymmetric mapping, corresponding to any initial vector field in the fiber of the tangent space to 
Teichmüller space $T$, we obtain two sections of the tangent bundle to $T$, one corresponding to earthquaking and one corresponding to bending. By Thurston's earthquake theorem [18], we know that the earthquake section is always integrable, but the corresponding problem for bending is unsolved. These topics are discussed in the appendix.

I would like to thank Dennis Sullivan for many helpful suggestions and discussions. Nikola Lakic has also made several important corrections and has provided the proof of the uniqueness part of Theorem 5.1.

\section{The TANgent SPaCe to TeichmülleR SPACE}

An orientation preserving mapping $h$ of an interval is quasisymmetric if there exists a constant $M$ such that

$$
\frac{1}{M} \leq \frac{h(x+t)-h(x)}{h(x)-h(x-t)} \leq M
$$

for all triples of points, $x-t, x$, and $x+t$ in the interval. It is quasisymmetric on the circle if, when viewed in projective coordinate charts mapping to intervals, it is quasisymmetric there. We let $Q S$ be the space of all such orientation preserving quasisymmetric mappings of a circle into itself. To define a topology on $Q S$ we declare that two homeomorphisms $h_{1}$ and $h_{2}$ are close to each other if they are close in the uniform topology and if $h_{2} \circ h_{1}^{-1}$ satisfies (1.1) with the constant $M$ near to 1 . It should satisfy (1.1) viewed in any projective coordinate chart selected from a finite number of such charts whose domains of definition cover the circle. It follows that, for any fixed quasisymmetric homeomorphism $g$, the right composition mapping $h \mapsto h \circ g$ is continuous. The neighborhoods of the identity in this topology are unaffected by postcomposition by a Möbius transformation which preserves the circle.

As a model for the circle with projective structure on which the elements of $Q S$ are defined, we usually take the extended real axis, $\overline{\mathbb{R}}$, or the unit circle, $S^{1}$, in the complex plane, both with the standard projective structure.

Universal Teichmüller space $T$ equals the coset space $Q S \bmod \operatorname{PSL}(2, \mathbb{R})$ with the quotient topology; two elements $h_{1}$ and $h_{2}$ are in the same coset if $A \circ h_{1}=h_{2}$ for some Möbius transformation $A$. The tangent space to $T$ is a certain Banach space of continuous vector fields $V(x) \frac{d}{d x}$ defined on $\overline{\mathbb{R}}$ factored by the three dimensional subspace of quadratic polynomials with real coefficients. This subspace corresponds to those vector fields which are tangent vectors to curves of Möbius transformations. To describe a norm for this Banach space, we pick an arbitrary set of three contiguous intervals $L, M, R$ on the circle and let $T$ be the union of these three intervals. $L, M, R$, and $T$ stand for left, middle, right, and total. We let these letters also stand for the directed lengths of these intervals. This means that if $L, M$, and $R$ are positive, then $T$ is negative. The ratio $M T / L R$ is one of the cross ratios of the four boundary points of these intervals. In general, a cross ratio of four distinct points takes values in $\overline{\mathbb{C}}-\{0,1, \infty\}$ and, if the four points lie on a circle or a line, the cross ratio is real. Let $\mathrm{cr}$ be the cross ratio $M T / L R$ and $\rho$ be the Poincaré metric for $\overline{\mathbb{C}}-\{0,1, \infty\}$. For any interval $J$, let

$$
V^{\prime}(J)=\frac{V(\text { right endpoint of } J)-V(\text { left endpoint of } J)}{\text { right endpoint of } J \text {-left endpoint of } J}
$$


and let the norm of $V$ be

$$
\|V\|_{\mathscr{Z}}=\sup \left|(\mathrm{cr}) \rho(\operatorname{cr})\left\{V^{\prime}(L)-V^{\prime}(M)+V^{\prime}(R)-V^{\prime}(T)\right\}\right|,
$$

where the supremum is taken over all possible choices of contiguous sets of three intervals $L, M, R$ with $T=L \cup M \cup R$. For every choice of $L, M, R$, and $T$, the curve $h_{t}(x)=x+t V(x)+o(t)$ induces a curve of cross ratios moving along the real axis in $\overline{\mathbb{C}}-\{0,1, \infty\}$. The assertion that $\|V\|_{\mathscr{I}}$ is finite amounts to saying that this curve has bounded instantaneous velocity at $t=0$ in the Poincaré metric. Notice that $\|V\|_{\mathscr{Z}}=0$ if, and only if, $V$ is a quadratic polynomial. Thus $\|V\|_{\mathscr{Z}}$ annihilates precisely those vector fields which are tangent vectors to curves of Möbius transformations.

Remark. We could use the same norm $\|V\|_{\mathscr{Z}}$ as a norm for complex-valued vector fields $V(z) \frac{d}{d z}$ defined in the extended complex plane $\overline{\mathbb{C}}$. Then it is an exercise to show that $\|V\|_{\mathscr{Z}}<\infty$ if, and only if, $|V(z)|=O\left(z^{2}\right)$ as $z \rightarrow \infty$ and $V$ has first partial derivatives in the generalized sense and $\bar{\partial} V=\mu$ is in $L_{\infty}(\mathbb{C})$. Moreover, $\|V\|_{\mathscr{Z}} \leq\|\mu\|_{\infty} \leq$ (const) $\|V\|_{\mathscr{Z}}$ and $V(z)$ differs by at most a quadratic polynomial in $z$ from the integral

$$
-\frac{1}{\pi} \iint_{\mathbb{C}} \mu(\zeta)\left\{\frac{z(z-1)}{\zeta(\zeta-1)(\zeta-z)}\right\} d \xi d \eta
$$

Since near $z=0$ the Poincare density $\rho(z)$ is asymptotic to $\left(|z| \log |z|^{-1}\right)^{-1}$, the appearance of the Poincare density $\rho$ in the definition (1.2) shows that a bound on $\|V\|_{\mathscr{I}}$ implies $V(z)$ has an $|\varepsilon \log \varepsilon|$-modulus of continuity.

If we let $L=[x-t, x], M=[x, x+t]$, and $R=[x+t, x+b]$, then as $b$ approaches $\infty$, the cross ratio $M T / L R$ of directed lengths approaches -1 and, assuming $V(x)=o\left(x^{2}\right)$ as $x$ approaches $\infty$, the expression in the supremum of (1.2) approaches the constant $\rho(-1)$ multiplied by

$$
\left|\frac{V(x+t)+V(x-t)-2 V(x)}{t}\right| \text {. }
$$

The space of continuous functions $V(x)$ for which (1.3) is bounded for all $x$ and $t$ is called the space of Zygmund bounded functions. In [20], Zygmund shows by direct calculation that a bound on (1.3) implies $V(x)$ satisfies an $|\varepsilon \log \varepsilon|$-modulus of continuity.

In [6] it is proved that the tangent space to Teichmüller space can be identified with the Banach space of continuous functions defined on $\mathbb{R}$ for which (1.3) is bounded factored by the two dimensional subspace of affine functions. A norm on this space of functions can be defined as the supremum of $\varepsilon(x, t)$ for all $x$ and $t$ in $\mathbb{R}$, where $\varepsilon(x, t)$ is given by (1.3). This norm is equivalent to the norm given by (1.2) on those $V(x)$ which grow no faster than $|x \log | x||$ as $|x| \rightarrow \infty$. Moreover, any element $V$ of $\mathscr{Z}$, after the subtraction of a suitable quadratic polynomial, is one for which $\varepsilon(x, t)$ is bounded.

The tangent space to Teichmüller has other descriptions. It is

(i) the dual Banach space to the space $\mathscr{A}$ of integrable holomorphic quadratic differentials defined in the upper half plane $\mathbb{H}$,

(ii) a factor space of $L_{\infty}$ complex-valued functions defined on $\mathbb{H}$, and

(iii) the space $\mathscr{B}$ of bounded cusp forms on $\mathbb{H}$. 
The space $\mathscr{A}$ consists of all complex-valued holomorphic functions $\varphi$ defined on $\mathbb{H}$ for which $\|\varphi\|_{\mathscr{A}}=\iint_{\mathbb{H}}|\varphi(z)| d x d y$ is finite. Any measurable, complexvalued function $\mu$ in $L_{\infty}$ and defined on $\mathbb{H}$ is paired with any element $\varphi$ in $\mathscr{A}$ by

$$
\langle\varphi, \mu\rangle=\operatorname{Re} \iint_{\mathbb{H}} \mu(z) \varphi(z) d x d y .
$$

We let $N$ be the subspace of $L_{\infty}$ consisting of those elements which annihilate $\mathscr{A}$ under this pairing. The space $\mathscr{B}$ of all bounded cusp forms consists of those functions $\psi(z)$ which are holomorphic in $\mathbb{H}$ and such that the metric $|\psi(z)|^{1 / 2}|d z|$ is bounded compared to the Poincare metric; in effect, this means that we take as the norm on $\psi$ the expression

$$
\|\psi\|_{\infty}=\sup \left|y^{2} \psi(z)\right|
$$

where $z=x+i y$ and the supremum is over all $z$ in $\mathbb{H}$. Theorem 1.1 is a summary of results appearing in several different places, [1, 3, 6, 12, 15]:

Theorem 1.1. $\mathscr{A}^{*}, L_{\infty} / N, \mathscr{Z}$, and $\mathscr{B}$ are isomorphic Banach spaces, each of them identifiable naturally with the tangent space to Teichmüller space.

We give here a description of the mappings which yield the isomorphisms. The isomorphism between $\mathscr{A}^{*}$ and $L_{\infty} / N$ comes from the pairing between $\mathscr{A}$ and $L_{\infty}$. By the Hahn-Banach theorem a linear functional on $\mathscr{A}$ extends to a linear functional on $L_{1}$ with the same norm and, by the Riesz representation theorem, a bounded linear functional on $L_{1}$ can be represented as an element of $L_{\infty}$. Two such representations correspond to the same element of $\mathscr{A}^{*}$ if their difference annihilates $\mathscr{A}$. To describe the isomorphism from $\mathscr{Z}$ and $L_{\infty} / N$, start with a real-valued function $V$ in $\mathscr{Z}$ and apply the BeurlingAhlfors extension formula to it to obtain a complex-valued function $\widehat{V}$ defined on $\mathbb{H}$. Any extension $\widehat{V}$ of $V$ with bounded $\bar{\partial}$-derivative would suffice. Let $\mu=\bar{\partial} \widehat{V}$. The mapping $V \mapsto \widehat{V} \mapsto \mu$ yields an isomorphism from $\mathscr{Z}$ onto $L_{\infty} / N$ (see [6]). To obtain the isomorphism from $\mathscr{Z}$ to $\mathscr{B}$, apply the Hilbert transform $H$ to $V . H V$ is determined, up to additive real constant, by the condition that $V(x)+i H V(x)$ has a holomorphic extension $V(z)+i H V(z)$ to the upper half plane. Let $\psi$ be the third derivative of this holomorphic function. The transformation $V \mapsto \psi$ is an isomorphism from $\mathscr{Z}$ onto $\mathscr{B}$.

Theorem 1.1 implies an assertion about smooth curves of quasisymmetric homeomorphisms passing through the identity. On the one hand, if $h_{t}$ is a $C^{1}$ curve in $Q S$ such that $h_{0}$ is the identity, then there exists a vector field $V(x) \frac{d}{d x}$ in $\mathscr{Z}$ such that

$$
h_{t}(x)=x+t V(x)+o(t)
$$

where the constant in $o(t)$ is uniform with respect to $x$ in bounded intervals. On the other hand, if a vector field $V(x) \frac{d}{d x}$ in $\mathscr{Z}$ is given, then there exists a real analytic curve $h_{t}$ in $Q S$ such that (1.5) holds.

Assume $\varphi$ in $\mathscr{A}$ is a rational function given by a finite sum

$$
\varphi(z)=\sum \frac{\rho_{j}}{z-x_{j}},
$$

where $\rho_{j}$ and $x_{j}$ are real, $\sum \rho_{j}=0$, and $\sum \rho_{j} x_{j}=0$. The last two conditions assure that $\|\varphi\|_{\mathscr{A}}=\iint_{\mathbb{H}}|\varphi(z)|<\infty$. By a theorem of Bers [12], the set of such 
rational functions, with simple poles at the points $x_{j}$ on the real axis and with real-valued residues $\rho_{j}$, is dense in $\mathscr{A}$.

Under the isomorphisms of Theorem 1.1, the pairing between $\mathscr{A}$ and $L_{\infty} / N$ corresponds to the pairing between $\mathscr{A}$ and $\mathscr{Z}$ given by

$$
\langle\varphi, V\rangle=-\frac{\pi}{2} \sum V\left(x_{j}\right) \rho_{j},
$$

where $\varphi$ is given by (1.6). If we define

$$
\|V\|_{T}=\sup |\langle\varphi, V\rangle|
$$

where the supremum is over all $\varphi$ of norm 1 in $\mathscr{A}$, we get a norm equivalent to the Zygmund norm of (1.4) (see [6]). Since it is known that

$$
\|\mu\|_{T}=\sup \left\{\left|\operatorname{Re} \iint \mu(z) \varphi(z) d x d y\right|: \varphi \text { of norm } 1\right\}
$$

gives the infinitesimal form of Teichmüller's metric on universal Teichmüller space [4, 12], we have

Theorem 1.2. The supremum (1.8) over all rational holomorphic quadratic differentials $\varphi$ of norm 1 gives the infinitesimal form of Teichmuller's metric on $\mathscr{Z}$, the tangent space to $Q S \bmod \operatorname{PSL}(2, \mathbb{R})$. In particular, $\|V\|_{\mathcal{Z}}$ defined in (1.2) and $\|V\|_{T}$ defined in (1.8) are equivalent norms on $\mathcal{Z}$.

We now give two examples of curves $h_{t}$ in $Q S$ with tangent vectors in $\mathscr{Z}$ which play a central rôle.

Example 1 (A simple earthquake). Let $E(x)=x$ for $x \geq 0$ and $E(x)=0$ for $x \leq 0$. Let

$$
h_{t}(x)= \begin{cases}e^{t} x & \text { for } x \geq 0 \\ x & \text { for } x \leq 0 .\end{cases}
$$

Then $h_{t}$ is a curve in $Q S$ passing through the identity with tangent vector $E(x)$ at $t=0$. Moreover,

$$
\frac{d}{d t} h_{t}(x)=E\left(h_{t}(x)\right)
$$

for every $x$ and $t$. A quasiconformal extension of $h_{t}$ to the upper half plane is

$$
w_{t}\left(r e^{i \theta}\right)=\left(e^{t(\pi-\theta) / \pi}\right) r e^{i \theta}
$$

and its dilatation $K$ satisfies $K+1 / K=2+(t / \pi)^{2}$.

Example 2 (A simple bending). Let $B(x)=x \log |x|$ and

$$
h_{t}(x)=|x|^{e^{t}} \operatorname{sign}(x) \text {. }
$$

Then $h_{t}$ is a curve in $Q S$ passing through the identity with tangent vector $B(x)$ at $t=0$. Moreover,

$$
\frac{d}{d t} h_{t}(x)=B\left(h_{t}(x)\right)
$$

for every $x$ and $t$. A quasiconformal extension of $h_{t}$ to the upper half plane is

$$
w_{t}\left(r e^{i \theta}\right)=r^{e^{t}} e^{i \theta},
$$

and its dilatation is $e^{t}$. 
The Teichmüller distance of a quasisymmetric mapping from the identity is equal to the infimum of the logarithms of the dilatations of all quasiconformal homeomorphisms which extend the given mapping to the upper half plane. Comparing Examples 1 and 2, we see that while the bending moves an unbounded distance away from the identity, the earthquake moves an amount which is on the order of the logarithm of that distance.

There is a simple relationship between the vector fields $E(x)$ and $B(x)$ in these two examples. The Hilbert transform of $E(x)$ is a constant times $B(x)$. This fact can be seen by looking at the real and imaginary parts of the function $z \log z$ which is holomorphic in the upper half plane. Using the polar notation $z=r e^{i \theta}$ and picking the branch of $\log z=\log r+i \theta$, where $\theta$ is between 0 and $\pi$, we have

$$
z \log z=(x+i y)(\log r+i \theta)=x \log r-y \theta+i(x \theta+y \log r) .
$$

Setting $y$ equal to zero, we find that the Hilbert transform of $x \log |x|$ is the function which is equal to 0 for positive values of $x$ and equal to $\pi x$ for negative values of $x$. Since the Hilbert transform is an involution, that is, since $H^{2}=-I$, the Hilbert transform of a bend is an earthquake and the transform of an earthquake is a bend.

\section{FOUR POINTS OF THE COMPASS}

We now construct two different curves of quasiconformal homeomorphisms of the upper half plane. To specify these quasiconformal mappings, we use the Beltrami equation

$$
w_{\bar{z}}(z)=\mu(z) w_{z}(z) .
$$

Given $\mu$ in $L_{\infty}(\mathbb{C})$ with $\|\mu\|_{\infty}<1$, this equation has a solution $w$ which is a quasiconformal homeomorphism of $\overline{\mathbb{C}}$ and which is unique up to postcomposition by a Möbius transformation [2]. Assume the Beltrami coefficient $\mu$ satisfies the symmetry condition, $\mu(\bar{z})=\overline{\mu(z)}$. For such $\mu$ the mapping $w$ preserves the real axis and its restriction to the real axis is quasisymmetric. If we assume $w$ fixes 0,1 , and $\infty$, then $w$ is determined by $\mu$. Let $\theta_{0}$ and $\theta_{1}$ be close to $\pi / 2$ with $\theta_{0}$ less than and $\theta_{1}$ greater than $\pi / 2$. Consider the sector $S$ in the upper half plane defined by $\theta_{0} \leq \arg z \leq \theta_{1}$. Let $S_{1}$ be the first quadrant minus this sector and $S_{2}$ be the second quadrant minus this sector. Define a mapping $w_{t}$, which coincides on the real axis with the mapping $h_{t}$ of Example 1, by

$$
w_{t}\left(r e^{i \theta}\right)=\left\{\begin{array}{l}
e^{t} r e^{i \theta} \quad \text { in } S_{1}, \\
e^{t\left(\theta_{1}-\theta\right) /\left(\theta_{1}-\theta_{0}\right)} r e^{i \theta} \text { in } S, \\
r e^{i \theta} \quad \text { in } S_{2} .
\end{array}\right.
$$

The Beltrami coefficient $\mu_{t}$ of $w_{t}$ is identically zero in $S_{1} \cup S_{2}$ and, in $S$, it is equal to

$$
\frac{t}{2 i\left(\theta_{1}-\theta_{0}\right)-t} \cdot \frac{z}{\bar{z}}
$$


Differentiating the Beltrami coefficient of $w_{t}$ at $t=0$ yields

$$
\left.\frac{d}{d t} \mu_{t}\right|_{t=0}=\left\{\begin{array}{l}
-\frac{i}{2\left(\theta_{1}-\theta_{0}\right)} \cdot \frac{z}{\bar{z}} \text { for } z \text { in } S, \\
0 \quad \text { elsewhere. }
\end{array}\right.
$$

Thus, as a vector field, this Beltrami coefficient represents the linear functional in $\mathscr{A}^{*}$ given by

$$
\varphi \mapsto-\operatorname{Re} \frac{i}{2\left(\theta_{1}-\theta_{0}\right)} \iint_{S} \varphi(z) \frac{z}{\bar{z}} d x d y .
$$

The curve of quasisymmetric mappings determined by the restriction of $w_{t}$ to the real axis is independent of the different choices of $\theta_{1}$ and $\theta_{0}$. So we can let $\theta_{1}$ decrease to $\pi / 2$ and $\theta_{0}$ increase to $\pi / 2$ without changing the vector field. The expression becomes

$$
\begin{aligned}
\varphi & \mapsto-\int_{0}^{\infty} \operatorname{limit}_{\theta_{0}, \theta_{1} \rightarrow \pi / 2} \operatorname{Re} \frac{i}{2\left(\theta_{1}-\theta_{0}\right)} \int_{\theta_{0}}^{\theta_{1}} \varphi\left(r e^{i \theta}\right) e^{2 i \theta} r d \theta d r \\
& =-\operatorname{Re} \frac{i}{2} \int_{0}^{i \infty} \varphi(z) z d z
\end{aligned}
$$

where the last integration is along the positive imaginary axis. This formula gives a bounded linear functional on $\mathscr{A}$ and, from formula (2.2), it is the infinitesimal form of a left twist. In the limit, the sector $S$ converges to the imaginary axis and the mapping converges to $z \mapsto e^{t} z$ in the first quadrant and the identity in the second quadrant. It is discontinuous on the imaginary axis, which is the fault line. It is a left earthquake because, looking from the second quadrant, the first quadrant appears to move to the left. If $t$ is replaced by $-t$ in the formula, we obtain a right earthquake so changing the sign on the right-hand side of (2.4) yields the infinitesimal form of a right earthquake.

Next, we consider a quasiconformal mapping which preserves the upper half plane, which has Beltrami coefficient supported in the sector $S$, and whose restriction to the real axis is the bending mapping of Example 2. It is unnecessary to give an explicit formula for this mapping. Instead, we merely specify a Beltrami coefficient which corresponds to fattening the sector $S$ to a thicker sector $S^{\prime}$. Consider the mapping from $S$ to $S^{\prime}$ given by $r e^{i \theta} \mapsto r e^{i K \theta}$. To make this into a curve of mappings which fattens by an amount which is independent of the size of the sector, we let $K=(1+k) /(1-k)$ and $k=t / 2\left(\theta_{1}-\theta_{0}\right)$. If $t$ is replaced by $-t$, we obtain a thinning deformation. The Beltrami coefficient of this mapping is

$$
\mu_{t}= \begin{cases}-k \cdot \frac{z}{z} & \text { in the sector } S \\ 0 & \text { elsewhere. }\end{cases}
$$

Extending $\mu_{t}$ to the whole plane by the symmetry condition $\mu(\bar{z})=\overline{\mu(z)}$ and solving the Beltrami equation (2.1), we obtain a quasiconformal homeomorphism $w_{t}$ of $\mathbb{C}$ which preserves the real axis. The derivative of $\mu_{t}$ with respect to $t$ and evaluated at $t=0$ is

$$
-\frac{1}{2\left(\theta_{1}-\theta_{0}\right)} \cdot \frac{z}{\bar{z}} \quad \text { in the sector } S \text { and zero elsewhere. }
$$


On comparing this formula with (2.3) and (2.4), we see that the linear functional on $\mathscr{A}$ which realizes an infinitesimal fattening of the imaginary axis is

$$
\varphi \mapsto-\operatorname{Re} \int_{0}^{i \infty} \varphi(z) z d z .
$$

Let $L$ be the complex linear functional on $\mathscr{A}$ given by $L(\varphi)=\int \varphi(z) z d z$ where the integral is over any arc in the upper half plane joining zero to $i \infty$. The results of (2.4) and (2.6) can be summarized as follows.

Theorem 2.1 (Four Points of the Compass). $\operatorname{Re} L, \operatorname{Im} L,-\operatorname{Re} L$, and $-\operatorname{Im} L$ represent, infinitesimally, thinning, left twisting, fattening, and right twisting, respectively, along the imaginary axis. If $a$ and $b$ are two points on the real axis, then the analogous statement is true with the imaginary axis replaced by the non-Euclidean line joining $a$ to $b$ and $L$ replaced by the complex linear functional

$$
\varphi \mapsto \int_{a}^{b} \varphi(z) \frac{(z-a)(z-b)}{a-b} d z .
$$

Here, the integration is along any arc in the upper half plane joining $a$ to $b$.

Proof. We change variables in (2.6) by the Möbius transformation $B(z)=$ $(z-a) /(b-z)$ in $\operatorname{PSL}(2, \mathbb{R})$. The vector field $z / d z$ transforms to $(z-a)(z-b) /((a-b) d z)$ and pairing this with the quadratic differential $\varphi(z) d z^{2}$ yields (2.7). Because the integrand is holomorphic in the upper half plane, the integration is independent of the path in the upper half plane which joins $a$ to $b$.

Notice that interchanging the letters $a$ and $b$ in formula (2.7) does not change its value. The functional $L$ depends only on the unoriented line in $\mathbb{H}$ joining $a$ to $b$.

The next goal is to show that the linear functionals described in this theorem coincide with the ones derived in Examples 1 and 2 of section 1. In effect, this means we must find the elements of $\mathscr{Z}$ which correspond under the isomorphism between (i) and (iii) in Theorem 1.1 to the linear functionals described in Theorem 2.1. Assume $\varphi$ is of the form (1.2); we must calculate $\operatorname{Re} \int z \varphi(z) d z$ where the integration is from 0 to $i \infty$. We get

$$
\begin{aligned}
\operatorname{Re} \int \sum \frac{z \rho_{j}}{z-x_{j}} d z & =\operatorname{Re} \int \sum\left(\frac{z \rho_{j}-x_{j} \rho_{j}}{z-x_{j}}+\frac{x_{j} \rho_{j}}{z-x_{j}}\right) d z \\
& =\operatorname{Re} \int \sum \frac{x_{j} \rho_{j}}{z-x_{j}} d z
\end{aligned}
$$

because $\sum \rho_{j}=0$. Here each of the integrals is from 0 to $i \infty$. A primitive in the upper half plane for the integrand on the right-hand side of $(2.8)$ is

$$
\sum x_{j} \rho_{j} \ln \left(z-x_{j}\right)
$$

where we take the single-valued branch of the logarithm in the upper half plane which has imaginary part between 0 and $\pi$. Since $\sum x_{j} \rho_{j}=0$, when we take the real part of this expression, the contribution at $i \infty$ is zero. We obtain

$$
\operatorname{Re} L(\varphi)=-\sum x_{j} \rho_{j} \ln \left|x_{j}\right|=\langle\varphi, V\rangle, \quad \text { where } V(x)=\frac{2}{\pi} x \ln |x| .
$$


This means that $\operatorname{Re} L$ coincides as a linear functional with $\frac{2}{\pi} B(x)$ from Example 2.

If we calculate the imaginary part of the integral in (2.8), once again we find the contribution from the upper limit of integration is zero. The lower limit contributes $-\pi \sum x_{j} \rho_{j}$ where the sum is over the terms $x_{j}>0$. This means $\operatorname{Im} L(\varphi)=\langle\varphi, 2 E\rangle$ where $E(x)$ is the vector field defined in Example 1 , tangent to a simple left twist along the imaginary axis. In summary, we have Theorem 2.2. The vector field $\pi E(x)$ from Example 1 corresponds to $\frac{\pi}{2} \operatorname{Im} L$ in $\mathscr{A}^{*}$ and the vector field $B(x)$ from Example 2 corresponds to $\frac{\pi}{2} \operatorname{Re} L$. Thus $E(x)$ corresponds to a left twisting across the imaginary axis and $B(x)$ corresponds to a thinning along the imaginary axis.

\section{The ZYgmund SPACE AND THE HilbeRT TRANSFORM}

Any Zygmund bounded vector field $V(x) \frac{d}{d x}$ defined on $\overline{\mathbb{R}}$ and normalized to vanish at 0,1 , and $\infty$ may be written as

$$
V(x)=-\frac{1}{\pi} \iint_{\mathbb{C}} \mu(\zeta) R(x, \zeta) d \xi d \eta,
$$

$\zeta=\xi+i \eta$, where $\mu$ in $L_{\infty}(\mathbb{C})$ satisfies the symmetry condition $\mu(\bar{z})=\overline{\mu(z)}$ and where

$$
R(x, \zeta)=\frac{x(x-1)}{\zeta(\zeta-1)(\zeta-x)}=\frac{1}{\zeta-x}-\frac{x}{\zeta-1}+\frac{x-1}{\zeta} .
$$

In formula (3.1) the variable $x$ can be replaced by the complex variable $z$, giving a function $V(z)$, real-valued when $z$ is real and such that $V_{\bar{z}}=\mu(z)$. The integral formula for the classical Hilbert transform of $V$ is

$$
H V(x)=\text { p.v. } \frac{1}{\pi} \int_{-\infty}^{\infty} \frac{V(\xi) d \xi}{\xi-x},
$$

where p.v. means principal value and the integration is along the real axis. We prefer to define the Hilbert transform $H V$ of $V$ as the principal value of the integral

$$
\begin{aligned}
H V(x) & =\frac{1}{\pi} \int_{-\infty}^{\infty} V(\xi)\left\{\frac{1}{\xi-x}-\frac{x}{\xi-1}+\frac{x-1}{\xi}\right\} d \xi \\
& =\frac{1}{\pi} \int_{-\infty}^{\infty} V(\zeta) R(x, \zeta) d \zeta,
\end{aligned}
$$

which differs from (3.3) by a linear polynomial in $x$. Thus, considered as an element of $\mathscr{Z}, H V(x)$ coincides with the classical Hilbert transform. Since $\bar{\partial} V(\zeta)=\mu(\zeta)$, an application of Stokes' formula to (3.4) yields

$$
\begin{aligned}
H V(x) & =\frac{2 i}{\pi} \iint_{U} \mu(\zeta) R(x, \zeta) d \xi d \eta+i V(x) \\
& =-\frac{2 i}{\pi} \iint_{L} \mu(\zeta) R(x, \zeta) d \xi d \eta-i V(x)
\end{aligned}
$$

where $\zeta=\xi+i \eta$ and where $U$ and $L$ denote the upper and lower half planes. If we define $\tilde{\mu}$ by

$$
\tilde{\mu}(z)= \begin{cases}-i \mu(z) & \text { in the upper half plane } \\ i \mu(z) & \text { in the lower half plane }\end{cases}
$$


then by adding the two integrals in (3.5) corresponding to integration over the upper and lower half planes, $H V(x)$ can be rewritten as

$$
H V(x)=-\frac{1}{\pi} \iint_{\mathbb{C}} \tilde{\mu}(\zeta) R(x, \zeta) d \xi d \eta
$$

From (3.6), $\tilde{\mu}$ is also in $L_{\infty}(\mathbb{C})$ and satisfies the symmetry condition. Although (3.7) is a singular integral, it is easier to handle than (3.3) because it does not involve a principal value. Moreover, the transform lifts to the mapping $\mu \mapsto \tilde{\mu}$ given by (3.6), which does not involve any integration at all. One can see why any smoothness property describable in terms of vanishing properties of $\mu$ which are preserved under $\mu \mapsto \tilde{\mu}$ is also preserved under the Hilbert transform. As an example, we observe that $V(x)$ is $C^{1+\alpha}, 0<\alpha<1$, in an interval and otherwise in $\mathscr{Z}$ if, and only if, $V(x)$ is representable in the form (3.7) with bounded $\mu$ and with $|\mu(z)| \leq O\left(y^{\alpha}\right)$ for $z$ approaching points of the interval. Therefore, the Hilbert transform of $V$ is also $C^{1+\alpha}$ in the same interval. We have proved the following theorem, which is due to Zygmund and Kerckhoff. A proof that the Hilbert transform gives the almost complex structure on the tangent space to Teichmüller spaces of Fuchsian groups corresponding to their complex structures appears in the paper of Nag and Verjovsky, [13].

Theorem 3.1. The Hilbert transform $H$ of (3.3) preserves the Banach space $\mathscr{Z}$, which is the tangent space to Teichmüller space. Moreover, under the natural projection of the $L_{\infty}$ to $\mathscr{Z}$ given in Theorem 1.1, H lifts to the mapping $\mu \mapsto \tilde{\mu}$.

Remark. Zygmund proved that the Hilbert transform preserves the space $\mathscr{Z}$ in [20]. Kerckhoff observed that the Hilbert transform gives the complex structure on Teichmüller space.

\section{THURSTON BOUNDED MEASURES}

Let $M$ be the set of unordered pairs of distinct points in $\overline{\mathbb{R}} . M$ can be identified with the set of hyperbolic lines in $\mathbb{H}$ by assigning to the unordered pair $\{a, b\}$ the hyperbolic line $L_{a b}$ which joins $a$ to $b$. Let $\sigma$ be an atomic measure on $M$ which assigns mass $\lambda_{a b}$ to a point $\{a, b\}$ and, for every hyperbolic disk $D$ in $\mathbb{H}$, let

$$
\operatorname{mass}_{\sigma}(D)=\sum\left|\lambda_{a b}\right|\left(\text { hyperbolic length of } L_{a b} \cap D\right),
$$

where the summation is over all $\{a, b\}$ in the support of $\sigma$. Let the Thurston norm of $\sigma$ be

$$
\|\sigma\|_{\mathrm{Th}}=\sup \operatorname{mass}_{\sigma}(D),
$$

where the supremum is over all hyperbolic disks $D$ of radius one. Define $\mathscr{M}$ to be the Banach space of weak star limits of such measures with bounded Thurston norm.

Associated to any pair $\{a, b\}$ there is a simple left twist and a simple bend corresponding to Examples 1 and 2 in section 1 . The vector fields $E$ and $B$ of those examples are associated with the points 0 and $\infty$. Let $a<b$ be two real numbers and $L(x)=(x-a) /(b-x) ; L$ is a Möbius transformation preserving the orientation of $\overline{\mathbb{R}}$, taking $a$ to 0 and $b$ to $\infty$, and taking the interval $[a, b]$ to the positive real axis. The transformed vector fields 
$\pi E_{a b}(x)=E(L(x)) / L^{\prime}(x)$ and $B_{a b}(x)=B(L(x)) / L^{\prime}(x)$ are given by

$$
E_{a b}(x)=\left\{\begin{array}{l}
0 \text { for } x \text { outside of }[a, b], \\
(x-a)(x-b) /(a-b) \text { for } a \leq x \leq b
\end{array}\right.
$$

and

$$
B_{a b}(x)=\frac{(x-a)(x-b)}{a-b} \ln \left|\frac{x-a}{x-b}\right| .
$$

Up to the addition of quadratic polynomials, $\pi E_{a b}(x)$ and $B_{a b}(x)$ are the real and imaginary parts, restricted to the real axis, of a single-valued branch of the function

$$
\frac{(z-a)(z-b)}{a-b} \ln \frac{a-z}{z-b},
$$

holomorphic in the upper half plane. Thus $B_{a b}$ is the Hilbert transform of $\pi E_{a b}$.

There are linear mappings on $\mathscr{M}$ defined by

$$
\begin{gathered}
B \sigma(z)=\iint B_{a b}(x) d \sigma(a, b), \\
E \sigma(z)=\pi \iint E_{a b}(x) d \sigma(a, b),
\end{gathered}
$$

and

$$
\Theta \sigma(z)=\iint \frac{(a-b)^{2}}{(z-a)^{2}(z-b)^{2}} d \sigma(a, b) .
$$

We have a commutative diagram, where $H$ and $I$ are isomorphisms:

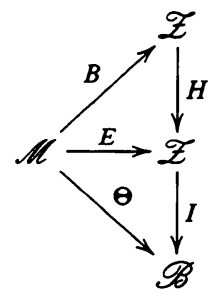

To show that any of the three mappings $B, E$, or $\theta$ is bounded, it suffices to show that $\theta$ is bounded. Since the discussion is invariant under conjugation by Möbius transformations, we may transform $\overline{\mathbb{R}}$ to the boundary of the unit circle and the upper half plane to its interior. Since the norms on $\mathscr{M}$ and $\mathscr{B}$ are invariant under the action of Möbius transformations, it suffices to estimate $\boldsymbol{\theta} \sigma(0)$ in terms of $\|\sigma\|_{\mathrm{Th}}$.

To obtain this estimate, we subdivide the unit disk by means of a Carleson grid. This means we cut the disk of Euclidean radius $1 / 2$ centered at the origin into two pieces by the diameter on the real axis. The annulus between this disk and the concentric circle of radius $3 / 4$ is cut by radial lines into four equal pieces. Denote this annulus by $A_{2}$. The annulus $A_{n}$ between $1-1 / 2^{n-1}$ and $1-1 / 2^{n}$ is cut into $2^{n}$ pieces by $2^{n}$ equally spaced radial lines. Note that there exists a number $C>0$ such that every piece of every annulus $A_{n}$ is contained in a non-Euclidean disk of radius $C$. 
Assume that $\sigma$ is an atomic measure; then the integral (4.5) is a summation and

$$
|\Theta \sigma(0)| \leq \sum|a-b|^{2}\left|\lambda_{a b}\right|
$$

where the summation is over all points $\{a, b\}$ in the support of $\sigma$. The denominator in the integrand of (4.5) drops out because we are assuming that the base space is the unit disk and, therefore, $|a|=|b|=1$. Let $S_{n}(\sigma)$ be the set of points $\{a, b\}$ in the support of $\sigma$ such that the hyperbolic line $L_{a b}$ enters the annulus $A_{n}$ but does not enter the annulus $A_{n-1}$.

We claim that the sum of $\left|\lambda_{a b}\right|$ over all $\{a, b\}$ in $S_{n}(\sigma)$ such that $L_{a b}$ enters one of the pieces of the annulus $A_{n}$ is bounded by a constant depending on $C$ and the Thurston norm of $\sigma$. If not, then the $\operatorname{mass}_{\sigma}(D)$ will not be bounded, where $D$ is a disk of radius $C$ and neither would it be bounded for disks of radius one. Since there are $2^{n}$ pieces in each annulus, it follows that

$$
\sum_{\{a, b\} \text { in } S_{n}(\sigma)}\left|\lambda_{a b}\right| \leq \text { (const) }\|\sigma\|_{\mathrm{Th}} 2^{n},
$$

where the constant depends on $C$. Since $\{a, b\}$ is in $S_{n}(\sigma)$, the line $L_{a b}$ does not enter the annulus $A_{n-1}$, which implies that $|a-b|<1 / 2^{n-1}$. Hence, the contribution in the summation (4.8) by the terms for which $\{a, b\}$ is in $S_{n}(\sigma)$ does not exceed a constant multiplied by $\|\sigma\|_{T h} 2^{n} / 2^{2 n-2} \mid$. Summing over $n$, we obtain a bound for the linear operator $\theta$.

Because $H$ and $I$ are isomorphisms, this argument proves the following theorem.

Theorem 4.1. The mappings $B, E$, and $\Theta$ in (4.7) are bounded linear mappings between Banach spaces.

\section{INFINITESIMAL EARTHQUAKE MEASURES}

The next objective is to show that each of the three mappings, $E, B$, and $\Theta$, in diagram (4.7) is surjective. Since $H$ and $I$ are isomorphisms, we can do this by exhibiting an inverse to $E$. We give a recipe for constructing a measure $\sigma_{V}$ in $\mathscr{M}$ associated with an element $V$ in $\mathscr{Z}$ such that $E\left(\sigma_{V}\right)=V$. The measure $\sigma_{V}$ is created by means of a quadratic polynomial convex hull construction.

From section 1 , we know that by subtracting a suitable quadratic polynomial from $V(x)$, we can make the vector field $V(x) \frac{d}{d x}$ vanish at $\infty$. This means that $|V(x)|$ grows no faster than a constant times $|x \log | x||$ as $|x|$ approaches $\infty$. After this preliminary adjustment, which has the effect of making $\infty$ a special point, we select any finite subset $S$ of $\overline{\mathbb{R}}$ which includes the point at $\infty$ and which contains at least four points. Our first objective is to construct a positive-valued atomic measure $\sigma_{S}$ which is determined solely by the values of $V$ restricted to $S$. The points of support of $\sigma_{S}$ are unordered pairs $\{a, b\}$ of distinct elements of $S$. We identify any unordered pair $\{a, b\}$ with the hyperbolic line joining $a$ to $b$. These lines are called lines of support for $\sigma_{S}$. The measure $\sigma_{S}$ will have the property that no two of its lines of support intersect.

Let $V_{S}$ equal $V$ restricted to $S$. A lower parabola for $V_{S}$ is a function $p(x)=A x^{2}+B x+C$, with $A<0$, whose graph lies on or below the graph of 
$V_{S}$ and which touches this graph at three or more points. We also allow $A=0$, but in that case the lower parabola is a lower line. We require that a lower line touch the graph of $V_{S}$ at two or more points and otherwise lie entirely on or below the graph of $V_{S}$. Because of the assumed growth property of $V(x) \frac{d}{d x}$ as $x$ approaches $\infty$, we can think of these lower lines as touching the graph of $V_{S}$ at three points, one of which is situated at $\infty$.

We divide the procedure for finding $\sigma_{S}$ into two parts, the linear and the quadratic. To begin the linear part we take a lower line $L_{1}$ for $V_{S}$ which touches its graph at its extreme left endpoint $x_{1}$ and at least at one other point $x_{2}$ to the right of $x_{1}$. We choose $x_{2}$ so that there is no other point to its right where $L_{1}$ touches the graph of $V_{S}$.

If there are no points of $S$ lying to the right of $x_{2}$ we go on to the quadratic part of the procedure. Assume for the moment that there is at least one other point of $S$ to the right of $x_{2}$. We then raise a lower line $L_{2}$ which touches the graph at the point above $x_{2}$ and at one other point to the right of $x_{2}$. We let $x_{3}$ be the $x$-coordinate of the point of $L_{2}$ which touches $V_{S}$ and is furthest to the right among all points of contact of $L_{2}$ with $V_{S}$. Then the unordered pair $\left\{x_{2}, \infty\right\}$ is in the support of $\sigma_{S}$ and it is assigned weight $\lambda_{2}$ equal to $\frac{1}{2}$ (the slope of $L_{2}$ - the slope of $L_{1}$ ). We continue this procedure to the right until there are no more points of $S$ lying further to the right. We obtain a finite sequence of unordered pairs $\left\{x_{2}, \infty\right\},\left\{x_{3}, \infty\right\},\left\{x_{4}, \infty\right\}, \ldots$ with weights $\lambda_{2}, \lambda_{3}, \lambda_{4}, \ldots$. Since they are all joined to $\infty$, none of the lines of support for these unordered pairs intersect.

Next, we describe the quadratic part of the procedure. It is applied separately, in each of the intervals subtended by points of contact with lower lines. If there is no point of $S$ between $x_{1}$ and $x_{2}$, we go on to the next interval. If there is at least one such point, we raise a lower parabola

$$
p_{1}(x)=\lambda_{a b} \frac{(x-a)(x-b)}{(a-b)}+L_{1}(x), \quad \lambda_{a b} \geq 0,
$$

where $\lambda_{a b}$ is chosen as large as possible so that $p(x)$ touches the graph of $V_{S}$ at least at one point between $a$ and $b$ and otherwise lies entirely below the graph of $V_{S}$. Then we assign weight $\lambda_{a b}$ to the unordered pair $\{a, b\}$ and note that the line of support for $\{a, b\}$ intersects none of the previously selected lines of support. After doing this, if possible, we repeat the quadratic procedure to the function $V_{S}(x)-p_{1}(x)$. The newly selected line of support intersects none of the previously selected lines of support and gives a new quadratic polynomial $p_{2}(x)$. We then apply the quadratic procedure to $V(x)-p_{1}(x)-p_{2}(x)$. When it is no longer possible, the procedure ends, and we obtain lines of support with endpoints $\left\{a_{j}, b_{j}\right\}$ where $a_{j}$ and $b_{j}$ are not adjacent points in $S$. Moreover, the weights $\lambda_{j}$ are such that, for $x$ in $S$,

$$
V_{S}(x)=\sum_{j} \lambda_{j} E_{a_{j} b_{j}}(x)+(\text { a quadratic polynomial }) .
$$

If we let $\sigma_{S}$ be the atomic measure with weights $\lambda_{j}$ at the support points $\left\{a_{j}, b_{j}\right\}$, we obtain $E\left(\sigma_{S}\right)=V_{S}$. We indicated how to construct the measure $\sigma$ of the following lemma.

Lemma 5.1. The measure $\sigma_{S}$ constructed above is nonnegative and has the nonintersection property. That is, the non-Euclidean lines of support $L_{a b}$ joining 
pairs of points $a$ and $b$, where $\{a, b\}$ is in the support of $\sigma_{S}$, do not intersect. No two adjacent pairs of points in $S$ are joined by a supporting line of $\sigma_{S}$. For values of $x$ in $S, E\left(\sigma_{S}\right)(x)$ differs from $V(x)$ by a quadratic polynomial. Moreover, $\sigma$ is the only atomic measure which is nonnegative, has the nonintersection property with lines of support joining nonadjacent pairs of points in $S$, and for which $E\left(\sigma_{S}\right)(x)$ differs from $V(x)$ by a quadratic polynomial.

Proof. To complete the proof of this lemma, we must show that $\sigma$ is uniquely determined. The construction of $\sigma$ can be obtained from the lower quadratic polynomial convex hull of the graph of $V$ restricted to $S$. A lower quadratic polynomial is any quadratic polynomial of the form $p(x)=A x^{2}+B x+C$, where $A \leq 0$. We define $p(x)$ to be allowable if, when $A<0$, its graph lies on or below the graph of $V$ restricted to $S$ and must touch $V$ restricted to $S$ at three or more points. When $A=0, p(x)$ is allowable if its graph lies on or below the graph of $V$ restricted to $S$ and it touches the graph of $V$ restricted to $S$ at two or more points. The lower quadratic polynomial hull of $V$ restricted to $S$, which we denote by hull $(V, S)$, is the intersection of regions lying above the graphs of these allowable functions $p(x)$. Notice that the shape of hull $(V, S)$ determines the lines of support for $\sigma_{S}$ and also the weights $\lambda_{j}$. If we find a piece of parabola bounding hull $(V, S)$ with endpoints at $a$ and $b$, then the line $L_{a b}$ joining $a$ to $b$ is a line of support of $\sigma$. Moreover, if $a$ is to the left of $b$ and if we let $V^{\prime}(a)$ denote the right derivative of $V$ at $a$ and $V^{\prime}(b)$ denote the left derivative of $V$ at $b$, then $\frac{1}{2}\left(V^{\prime}(a)-V^{\prime}(b)\right)=\lambda$ is the weight which is assigned to $L_{a b}$. Since $V$ restricted to $S$ uniquely determines $\operatorname{hull}(V, S)$, it follows that $\operatorname{hull}(V, S)$ uniquely determines $\sigma$.

Remark 1. Let $n$ be the number of points in $S$. If all of the nonnegative weights determined in the construction of $\sigma_{S}$ are positive, the measure $\sigma_{S}$ will have a system of $n-3$ lines of support. This system will be allowable in the following sense. No two of its lines intersect and no two adjacent points of $S$ are joined by a line. If all of the weights are positive, the system is maximal; it is not possible to add any more lines connecting points of $S$ and still have an allowable system. The number of allowable systems there are for $n=4,5$, and 6 is 2,5 , and 14 and, in general, for $n \geq 4$ it is the Catalan number

$$
N=\frac{1}{n-1}\left(\begin{array}{c}
2 n-4 \\
n-2
\end{array}\right) \text {. }
$$

Remark 2. For a finite set $S$ contained in the boundary of the unit circle and a homeomorphism of the boundary restricted to $S$, mapping $S$ to $h(S)$, there is a global form of the construction used to prove Lemma 5.1. This global result is described in the introduction. It involves arguing with $\operatorname{PSL}(2, \mathbb{R})$ instead of with quadratic polynomials. The construction gives a way of viewing the Teichmüller space for the configuration of the points of $S$ moving along the boundary of the unit circle as a topological cell. The cell is an amalgamation of $N$ different copies of $\mathbb{R}_{+}^{n-3}$, where $N$ is the Catalan number defined in the previous remark. These copies are sewn together according to the way the allowable configurations collapse into one another. To each cyclic order preserving mapping of the set $S$ to another subset $h(S)$ of $S^{1}$, determined up to postcomposition by a Möbius transformation, the construction gives a unique system of nonintersecting lines and a unique set of positive weights 
assigned to these lines. No two adjacent points of $S$ are joined by a line. If the system of lines is not maximal, it is on the boundary of two or more of the $N$ cells. As $n$ increases and $S$ becomes more and more dense in the circle, the complex of these $N$ cells becomes a better and better approximation to universal Teichmüller space.

Lemma 5.2. For a given vector field $V(x) \frac{d}{d x}$ in $\mathscr{Z}$, the measures $\sigma_{S}$ constructed from $V$ restricted to the set $S$ have Thurston norm bounded independently of the set $S$ or the number $n$ of elements in $S$.

Proof. Since each $\sigma_{S}$ is a nonnegative measure, it suffices to show that for bounded closed intervals $L, M, R$, and $T=L \cup M \cup R$ in $\overline{\mathbb{R}}$ for which the cross ratio $M T / L R$ is equal to -1 , the mass $\sigma_{S}(L \times R)$ is bounded independently of $S$. From Theorem 1.2, we can use the Zygmund norm and the Teichmüller norm interchangeably. Because the Teichmüller norm on an element $V(x) \frac{d}{d x}$ in $\mathscr{Z}$ is a Möbius invariant, we may choose a coordinate so that $L$ is an interval on the negative real axis and so that $R$ is an interval on the positive real axis. Let $P_{1}$ be the element of $S$ which is contained in $L$ and which is nearest to zero. If there is no such element, then $\sigma_{S}(L \times R)=0$. Similarly, let $P_{2}$ be the element of $S$ which is contained in $R$ and which is nearest to zero. If we delete from $L$ the interval $\left[P_{1}, 0\right]$ and from $R$ the interval $\left[0, P_{2}\right]$, we do not change the mass $\sigma_{S}(L \times R)$. We assume that $L$ and $R$ have been altered in this manner. Since $P_{1}$ is joined to $P_{2}$ by a line of support for $\sigma_{S}$, there must be an element of $S$ lying between $P_{1}$ and $P_{2}$. By an affine change of coordinate, not changing the Teichmüller norm of $V$, we can assume that 0 is this element of $S$, that $P_{1} \leq-1$, and that $P_{2} \geq 1$. The same type of argument allows us to assume that $L$ is bounded below and that $R$ is bounded above. By adding a linear function to $V$, not changing the Zygmund norm of $V$, we can assume that $V\left(P_{1}\right)=V\left(P_{2}\right)=0$. The bound on the Zygmund norm of $V$ in this coordinate determines a bound $M$ on $V(0)$ depending on the positions of the endpoints of $L$ and $R$ and on $\|V\|_{\mathscr{Z}}$. Any parabola which passes through $L$ at a point with slope 1 and passes through $R$ at a point with slope -1 must have height $\geq 1 / 2$ above the point $x=0$. Therefore, the sum of the weights of the lines of support for $\sigma_{S}$ which join $L$ to $R$ is less than $2 M$ and this proves the lemma.

Now we select an ascending sequence $S_{1} \subset S_{2} \subset S_{3} \subset \cdots$ of finite subsets of $\overline{\mathbb{R}}$ such that the closure of the union of the $S_{n}$ 's equals $\overline{\mathbb{R}}$. We also select a sequence of intervals $I_{k}$ and $J_{k}$, with $I_{k}$ disjoint from $J_{k}$, such that the four endpoints of $I_{k}$ and $J_{k}$ have standard cross ratio equal to -1 and such that the union over $k$ of $I_{k} \times J_{k}$ covers $\overline{\mathbb{R}} \times \overline{\mathbb{R}}-$ \{diagonal $\}$. Then we can select a subsequence of the measures $\sigma_{n}$ associated with the sets $S_{n}$ which converge weakly to a nonnegative measure $\sigma$ on each $I_{k} \times J_{k}$. Since the mass of $\sigma$ over $I_{k} \times J_{k}$ cannot be greater than the limit superior over $n$ of the masses $\sigma_{n}\left(I_{k} \times J_{k}\right)$, the Thurston norm of $\sigma$ is bounded.

Lemma 5.3. Any weak limit $\sigma$ of the measures $\sigma_{n}$ is a Thurston bounded, nonnegative measure which does not have intersecting lines of support.

Proof. We have already proved the first part. If $\sigma$ does have intersecting lines of support, we may suppose two such lines have endpoints $\{a, b\}$ and $\{c, d\}$ and we may select four disjoint open intervals $A, B, C$, and $D$ containing 
$a, b, c$, and $d$, respectively. By applying the weak convergence of the measures $\sigma_{n}$ to $\sigma$ to integration against the characteristic functions of $A \times B$ and $C \times$ $D$, we would find that, for some value of $n$, the measure $\sigma_{n}$ would have intersecting lines of support. But this contradicts the nonintersection property of measures $\sigma_{n}$.

If $\sigma$ is any weak limit of $\sigma_{n}$ and $W(x)=\iint E_{a b}(x) d \sigma(a, b)$, then, since $\sigma$ is bounded in the Thurston norm, it follows from Theorem 4.1 that $W(x)$ is in $\mathscr{Z}$.

Lemma 5.4. Let $V(x)$ be an element of $\mathscr{Z}$ and let there be given an ascending chain of finite subsets $S_{1} \subset S_{2} \subset S_{3} \subset \cdots$ of $\overline{\mathbb{R}}$ containing the point at infinity whose union is dense in $\overline{\mathbb{R}}$. Let $\sigma_{n}$ be the measures constructed as in Lemma 5.1 from $V(x)$ restricted to $S_{n}$ and $\sigma$ a weak limit of $\sigma_{n}$ and

$$
W(x)=\iint E_{a b}(x) d \sigma(a, b) .
$$

Then $W(x)$ and $V(x)$ are identical elements of $\mathscr{Z}$.

Proof. We begin by remarking that $V_{n}(x)=\iint E_{a b}(x) d \sigma_{n}(a, b)$ coincides with $V(x)$ on $S_{n}$ and converges to $V$. This is because, on any compact set, the family of functions $V_{n}$ is equicontinuous with an $x \log x$ modulus of continuity, the constant for which depends on the uniform bound $\left\|V_{n}\right\|_{\mathscr{I}}$. Since for fixed $x, E_{a b}(x)$ is a continuous function of $a$ and $b$, we could conclude that $V(x)=W(x)$ if we knew that the sequence of measures $\sigma_{n}$ had compact support.

For contiguous intervals $L, M$, and $R$ with $T=L \cup M \cup R$, in the notation of (1.2) define $V(L M R T)$ to be $V^{\prime}(L)-V^{\prime}(M)+V^{\prime}(R)-V^{\prime}(T)$. Then $P(L M R T)=0$ for every choice of $L, M, R$ and $T$ if, and only if, $P$ is a quadratic polynomial. From the definition of the norm on $\mathscr{Z}$, our goal is to show that $V(L M R T)=W(L M R T)$ for every choice of $L, M, R$ and $T$. In calculating $W(L M R T)$, any set $I \times J$ in the support of $\sigma$, with $I$ to the left of $x_{1}$ and $J$ to the right of $x_{4}$, may be neglected from the integration because it contributes a function whose restriction to $T$ is a quadratic polynomial and, therefore, does not affect the evaluation of $W(L M R T)$. A similar remark applies to lines in the support of $\sigma$ both of whose endpoints are in the same interval, $L, M$, or $R$. Thus, to evaluate $W(L M R T)$, we only need to consider lines in the support of $\sigma$ which join $L$ to $M, L$ to $R$, or $R$ to $M$. Lines which join $L$ to $R$ form a compact set and on this set we apply the weak convergence of $\sigma_{n}$ to $\sigma$.

Now consider an interval $I$ of length $\varepsilon$ whose midpoint is $x_{2}$. We can estimate the amount that the points of support of $\sigma$ which join $L$ to $R$ and whose endpoints are both in $I$ contribute to $W(x)$. Because of formula (4.2) for $E_{a b}(x)$ and because of the bound $B$ on the Thurston norm, it can contribute no more that $B \varepsilon$ to the value of $W(x)$. But on $(L-I) \times(M-I), \sigma_{n}$ is converging weakly to $\sigma$. We can apply this same argument to the point $x_{3}$ and to the uniformly Zygmund bounded functions $V_{n}(x)$. In the end we obtain the desired conclusion, which is that $V(L M R T)=W(L M R T)$.

Theorem 5.1 (Gardiner and Lakic). $B$ and $E$ are bounded surjective linear operators from $\mathscr{M}$ to $\mathscr{Z}$ and $\Theta$ is a bounded surjective linear operator from $\mathscr{M}$ to the Banach space $B$ of bounded cusp forms. Moreover, the measure $\sigma_{V}$ 
is nonnegative, and it has nonintersecting lines of support. Furthermore, any measure $\sigma$ which is nonnegative, has nonintersecting lines of support, and is such that $E(\sigma)$ differs from $V$ by a quadratic polynomial must be identical to $\sigma_{V}$.

Remark. I am grateful to Nikola Lakic for filling in the uniqueness part of the proof of this theorem.

Proof. The first part of this theorem is a repeat of Theorem 4.1 with the additional fact that $B$ is surjective. But from Lemmas 5.3 and 5.4, given a $V$, we have constructed a nonnegative measure $\sigma$ with nonintersecting lines of support such that $E(\sigma)$ differs from $V$ by a quadratic polynomial. Now suppose that $\sigma_{1}$ and $\sigma_{2}$ are nonnegative, individually have nonintersecting lines of support and $E\left(\sigma_{1}\right)=V_{1}$ and $E\left(\sigma_{2}\right)=V_{2}$ are identical as elements of $\mathscr{Z}$. By adding a different quadratic polynomial to each one of them, we may assume that both of them are $O(|x \log | x||)$ as $x$ approaches $\infty$.

First, we claim that no support line of $\sigma_{1}$ can intersect a support line of $\sigma_{2}$. Assuming the contrary, suppose a support line $L_{1}$ of $\sigma_{1}$ meets the $x$ axis at $x_{1}$ and $x_{3}$ and a support line $L_{2}$ of $\sigma_{2}$ meets the $x$-axis at $x_{2}$ and $x_{4}$. Let $L=\left[x_{1}, x_{2}\right], M=\left[x_{2}, x_{3}\right]$, and $R=\left[x_{3}, \infty\right]$. By the growth condition on $V_{1}, V_{1}(L M R T)$ equals $V^{\prime}(L)-V^{\prime}(M)$. Since no support lines of $\sigma_{1}$ meet $L_{1}, V_{1}^{\prime}(L)-V_{1}^{\prime}(M)>0$. On the other hand, since no support line of $\sigma_{2}$ meets $L_{2}, V_{2}^{\prime}(L)-V_{2}^{\prime}(M)<0$. This contradicts the assumption that $V_{1}(L M R T)=V_{2}(L M R T)$ for all quadruples $L, M, R$, and $T$.

Second, we claim that $\sigma_{1}$ and $\sigma_{2}$ are identical measures. Let $\mathscr{L}$ and $\widetilde{L}$ be lines of support of $\sigma_{1}$ and assume $\mathscr{L}$ meets the real axis at two points $a_{0}$ and $b_{0}$ and $\widetilde{\mathscr{L}}$ meets the real axis at two points $a_{1}$ and $b_{1}$, with $a_{0}<a_{1}<b_{1}<b_{0}$. By a Möbius change of coordinates, we may assume that $a_{0}=0$ and $b_{0}=\infty$ and $\mathscr{L}$ is the imaginary axis. Because $a_{0}$ and $b_{0}$ now have specific values, we can simplify notation by letting $a_{1}=a$ and $b_{1}=b$. Let $I=[0, a]$ and $J=[b, \infty]$ so that these intervals together with $\mathscr{L}$ and $\widetilde{\mathscr{L}}$ enclose a region $D$. Let $t, 0<t<1$, be a constant multiple of the arclength parameter along the segment which is the common perpendicular to $\mathscr{L}$ and $\widetilde{\mathscr{L}}$, so that $t=0$ corresponds to the point on $\mathscr{L}$ and $t=1$ corresponds to the point on $\widetilde{\mathscr{L}}$. Each line of support passing through this common perpendicular segment meets the interval $[0, a]$ at some point $a(t)$ and meets the interval $[b, \infty]$ at some point $b(t)$. Some points of $[0, a]$ and of $[b, \infty]$ may not be endpoints of lines of support of $\sigma_{1}$ or of $\sigma_{2}$. But we may fill in nonintersecting non-Euclidean lines with endpoints at $a_{t}$ and $b_{t}$ so that to each point $t$ on the common perpendicular there corresponds a unique pair $\left(a_{t}, b_{t}\right)$ in $[0, a] \times[b, \infty]$. Let $\alpha_{j}(t)=\sigma_{j}\left(\left[0, a_{t}\right) \times\left(b_{t}, \infty\right]\right)$ for $j=1$ and 2. To show that the measures $\sigma_{j}$ are determined by the vector fields $V_{j}(x) \frac{d}{d x}$, it suffices to show that the monotonic functions $\alpha_{j}(t)$ are determined.

Without loss of generality, we may assume that $V_{1}(x) \frac{d}{d x}$ and $V_{2}(x) \frac{d}{d x}$ vanish at $\infty$. We are going to evaluate these vector fields at quadruples of points in the support of $\sigma_{1} \vee \sigma_{2}$. We will only consider quadruples two of whose points are in $[0, a]$ and two of whose points are in $[b, \infty]$. Moreover, all points of evaluation lie at endpoints of lines of support of $\sigma_{1} \vee \sigma_{2}$. We call such quadruples of points allowable quadruples. 
For the purposes of evaluating $V(L M R T)$ on allowable quadruples, we note that lines of support of $\sigma_{j}$ to the left of 0 do not affect $V_{j}(x)$ for $x>0$. Moreover, lines of support with both endpoints in $[0, a]$ or with both endpoints on $[b, \infty]$ do not affect the values of $V_{j}(L M R T)$ for allowable quadruples.

Letting $\sigma=\sigma_{1}-\sigma_{2}, V=V_{1}-V_{2}$, and $\alpha=\alpha_{1}-\alpha_{2}$ and applying the condition $V(L M R T)=0$ to intervals $L=\left[a_{s}, a_{r}\right], M=\left[a_{r}, b_{r}\right], R=\left[b_{r}, \infty\right]$ yield

$$
\frac{V\left(a_{r}\right)-V\left(a_{r}\right)}{a_{r}-a_{s}}-\frac{V\left(b_{r}\right)-V\left(a_{r}\right)}{b_{r}-a_{r}}=0 \text {. }
$$

Multiplying both sides by $\left(a_{r}-a_{s}\right)\left(b_{r}-a_{r}\right)$, observing that

$$
V\left(a_{t_{0}}\right)=\int_{0}^{t_{0}}\left(a_{t_{0}}-a_{t}\right)\left(a_{t_{0}}-b_{t}\right) \frac{d \alpha(t)}{a_{t}-b_{t}}
$$

and using the notation $d \eta(t)=d \alpha(t) /\left(a_{t}-b_{t}\right)$, we get

$$
\begin{gathered}
\left(b_{r}-a_{r}\right) \int_{0}^{s}\left(a_{s}-b_{t}\right)\left(a_{s}-a_{t}\right) d \eta(t)+\left(a_{r}-a_{s}\right) \int_{0}^{r}\left(b_{r}-a_{t}\right)\left(b_{r}-b_{t}\right) d \eta(t) \\
=\left(b_{r}-a_{s}\right) \int_{0}^{r}\left(a_{r}-a_{t}\right)\left(a_{r}-b_{t}\right) d \eta(t) .
\end{gathered}
$$

After a simplification and cancelling a common factor of $\left(b_{r}-a_{r}\right)$, we obtain $\int_{0}^{s}\left(a_{s}-a_{t}\right)\left(a_{s}-b_{t}\right) d \eta(t)-a_{s} \int_{0}^{r}\left(b_{r}+a_{r}-b_{t}-a_{t}\right) d \eta(t)=\int_{0}^{r}\left(b_{t} a_{t}-b_{r} a_{r}\right) d \eta(t)$.

On putting $s=0$, we get

$$
\int_{0}^{r}\left(b_{t} a_{t}-b_{r} a_{r}\right) d \eta(t)=0
$$

and

$$
\int_{0}^{s}\left(a_{s}-a_{t}\right)\left(a_{s}-b_{t}\right) d \eta(t)=a_{s} \int_{0}^{r}\left(b_{r}+a_{r}-b_{t}-a_{t}\right) d \eta(t) .
$$

By expanding out equation (ii) and using the equality

$$
\int_{0}^{s} a_{t} b_{t} d \eta(t)=a_{s} b_{s} \int_{0}^{s} d \eta(t)
$$

we obtain

$$
\int_{0}^{s}\left(a_{s}+b_{s}-a_{t}-b_{t}\right) d \eta(t)=\int_{0}^{r}\left(a_{r}+b_{r}-a_{t}-b_{t}\right) d \eta(t)
$$

and, therefore, this last integral is independent of $s$. By letting $s \rightarrow 0$, we see that, for all $r$,

$$
\int_{0}^{r}\left(b_{t}+a_{r}-b_{r}-a_{t}\right) d \eta(t)=0 .
$$

On each interval $[r, 1]$, with $r>0$, the functions $a_{t}$ and $b_{t}$ are continuous, monotone, and have bounded variation. Therefore, the functions $a_{t}+b_{t}$ and $a_{t} b_{t}$ also have bounded variation and there are measures $S$ and $P$ on the open interval $(0,1)$ such that for each half open interval $[x, y)$, with $0<x<y<$ $1, S([x, y))=a_{x}+b_{x}-a_{y}-b_{y}$ and $P([x, y))=a_{x} b_{x}-a_{y} b_{y}$. If we define 
$S(\{1\})=a_{1}+b_{1}=a+b$ and $P(\{1\})=a_{1} b_{1}=a b$, we see that, for all $t$ with $0<t<1$,

$$
a_{t}+b_{t}=\int_{t}^{1} d S(s) \text { and } a_{t} b_{t}=\int_{t}^{1} d P(s) .
$$

Using (iii), we obtain

$$
\int_{r}^{1} d S(s) \int_{0}^{r} d \eta(t)=\left(a_{r}+b_{r}\right) \int_{0}^{r} d \eta(t)=\int_{0}^{r}\left(a_{t}+b_{t}\right) d \eta(t)=\int_{0}^{r} \int_{t}^{1} d S(s) d \eta(t)
$$

which is equal by Fubini's theorem to

$$
\int_{0}^{r} d S(s) \int_{0}^{s} d \eta(t)+\int_{r}^{1} d S(s) \int_{0}^{r} d \eta(t)
$$

On letting $G(s)=\int_{0}^{s} d \eta(t)$, where by this notation we mean the integral over the interval $(0, s]$, we obtain $\int_{0}^{s} G(s) d S(s)=0$ for each $r$, and by repeating the same argument, using (i) instead of (iii), we also obtain $\int_{0}^{r} G(s) d P(s)=0$. Since $P$ and $S$ are Borel measures, these last two equations mean that $G(s)=0$ for almost all $s$ with respect to the measures $P$ and $S$. If for some value of $x, G(x)$ is not equal to zero, then since $G$ is right-continuous there is a value of $y>x$ so that $G(t)$ is not equal to zero on the interval $[x, y]$. Then, on that interval, the continuous functions $a_{t}+b_{t}$ and $a_{t} b_{t}$ must be constant. Since $\left(b_{t}-a_{t}\right)^{2}=\left(b_{t}+a_{t}\right)^{2}-4 b_{t} a_{t}$, we find that $b_{t}-a_{t}$ is constant on the interval $[x, y]$, which is a contradiction because $b_{t}-a_{t}$ is strictly decreasing for $0 \leq t \leq 1$.

Remark 1. Starting with the vector field $V(x) \frac{d}{d x}$, a natural expression to consider as an inverse to $B$ is the infinitesimal Schwarzian derivative of the curve of quasisymmetric mappings $h_{t}(x)=x+t V(x)+o(t)$. The expression for this infinitesimal derivative is

$$
\frac{\partial^{2}}{\partial x \partial y} \frac{V(x)-V(y)}{x-y} d x d y .
$$

This formula gives a formal inverse to $B$ (see [5]), but it is not a measure. It is only a distribution, giving a continuous linear functional on the space of Hölder continuous functions.

Remark 2. An alternative construction of the measure $\sigma_{V}$ is given in [10]. But the construction there does not deal with the Banach space bounds and does not follow an argument where one first constructs the measure for a vector field defined only on a finite set. The argument given here may have practical applications where the vector field comes from experimental or computational data and is, in fact, given only on a finite set.

\section{APPENDIX}

We pose here the problem of solving two differential equations, one corresponding to earthquaking (left twisting or right twisting) and the other corresponding to bending (fattening or thinning). We hope to give a method for solving these equations in joint work with Dennis Sullivan.

Given a measure $\sigma$ on $S^{1} \times S^{1}$ and a homeomorphism $H$ of $S^{1}$, let $h^{*} \sigma$ be the measure which assigns mass $\sigma\left(h^{-1}(T)\right)$ to any measurable set $T$. 
Lemma. If $\sigma$ is a Thurston bounded measure and $h$ is quasisymmetric, then $h^{*} \sigma$ is a Thurston bounded measure and there exist constants $A, B$, and $K(h)$ such that

$$
\left\|h^{*} \sigma\right\|_{\mathrm{Th}} \leq A K(h)\|\sigma\|_{\mathrm{Th}}+B
$$

where $K(h)$ is a constant depending on the size of $h$ in the quasisymmetric topology. The ordinary differential equation for earthquakes (left twisting or right twisting).

Let $\sigma=\sigma_{V}$ be the Thurston bounded measure corresponding to $V$ constructed in section 5. For this measure $\sigma$, let

$$
E_{h}(x)=\iint E_{a b}(x) d h^{*} \sigma(a, b) .
$$

This means that $E_{h}(x)$ is the image of $h^{*} \sigma$ under the map $E$ in (4.7). Find a curve of quasisymmetric mappings $h_{t}$ defined for all $t \geq 0$ with $h_{0}(x)=x$ and satisfying

$$
\left.\frac{d}{d s} h_{s+t} \circ h_{t}^{-1}(x)\right|_{s=0}=E_{h}(x) \text {. }
$$

This is the equation for left twisting; the equation for right twisting is obtained by changing the construction in section 5, using upper parabolas instead of lower parabolas. We already know that this equation has global solutions because of Thurston's earthquake theorem [18] and the application of that theorem to the class of quasisymmetric mappings [6].

The ordinary differential equation for bending (fattening or thinning).

Given a Zygmund bounded function $B(x)$, we find a measure $\sigma$ such that

$$
B(x)=\iint B_{a b}(x) d \sigma(a, b)
$$

where $B_{a b}$ are simple fattenings along the hyperbolic lines joining $a$ to $b$. For every $h$ in $Q S$, there are the measure $h^{*} \sigma$ and a Zygmund bounded function $B_{h}$ given by

$$
B_{h}(x)=\iint B_{a b}(x) d h^{*} \sigma(a, b) .
$$

This means that $B_{h}(x)$ is the image of $h^{*} \sigma$ under the map $B$ in (4.7). Find a curve of quasisymmetric mappings $h_{t}$ defined for all $t \geq 0$ with $h_{0}(x)=x$ and satisfying

$$
\left.\frac{d}{d s} h_{s+t} \circ h_{t}^{-1}(x)\right|_{s=0}=B_{h}(x) .
$$

\section{REFERENCES}

1. L. V. Ahlfors, Finitely generated Kleinian groups, Amer. J. Math. 86 (1964), 413-429.

2. L. V. Ahlfors and L. Bers, Riemann's mapping theorem for variable metrics, Ann. of Math. (2) 72 (1960), 385-404.

3. L. Bers, A non-standard integral equation with applications to quasiconformal mappings, Acta Math. 116 (1966), 113-134.

4. F. P. Gardiner, Teichmüller theory and quadratic differentials, Wiley-Interscience, 1987.

5. $\ldots$ correspondence between laminations and quadratic differentials, Complex Analysis Theory Appl. 6 (1986), 363-375. 
6. F. P. Gardiner and D. P. Sullivan, Symmetric structures on a closed curve, Amer. J. Math. 114 (1992), 683-736.

7. L Lacunary series as quadratic differentials in conformal dynamics, Contemporary Math., vol. 169, Amer. Math. Soc., Providence, RI, 1994, pp. 307-330.

8. John B. Garnett, Bounded analytic functions, Academic Press, 1981.

9. Oliver A. Goodman, Metrized laminations and quasisymmetric maps, Ph.D. thesis, Warwick University, 1989.

10. Paul Green, Vector fields and Thurston's theory of earthquakes, Ph.D. thesis, Warwick University, 1987.

11. S. Kerckhoff, The Nielsen realization problem, Ann. of Math. (2) 117 (1983), 235-265.

12. I. Kra, Automorphic forms and Kleinian groups, Benjamin, Reading, Mass., 1972.

13. S. Nag and A. Verjovsky, Diff( $\left.S^{1}\right)$ and the Teichmueller spaces, Comm. Math. Phys. 130 (1990), 123-138.

14. E. Reich and $\mathrm{K}$. Strebel, Extremal quasiconformal mappings with given boundary values, Contributions to Analysis (L. Ahlfors, I. Kra, B. Maskit, and L. Nirenberg, eds.), Academic Press, New York, 1974, pp. 375-392.

15. M. Riemann, Ordinary differential equations and quasiconformal mappings, Invent. Math. 33 (1976), 247-270.

16. D. Sullivan, Bounds, quadratic differentials and renormalization conjectures, Mathematics into the Twenty-first Century. II, Amer. Math. Soc., Providence, RI, 1992.

17. Luasiconformal homeomorphisms and dynamics. I, Solution of the Fatou-Julia problem on wandering domains, Ann. of Math. (2) 122 (1985), 401-418.

18. W. P. Thurston, Earthquakes in two-dimensional hyperbolic geometry, Low Dimensional Topology and Kleinian Groups, London Math. Soc. Lecture Note Series, vol. 112, 1984, pp. 91-112.

19. Scott Wolpert, The Fenchel-Nielsen deformation, Ann. of Math. (2) 115 (1982), 501-528.

20. A. Zygmund, Smooth functions, Duke Math. J. 12 (1945), 47-76.

Graduate Center (CUNY), 33 W. 42nd Street, New York, New York 10036-8099 